\title{
Escolaridade e padrões de ganho de peso na vida adulta no Brasil: Estudo Pró-Saúde
}

\author{
Maria de Jesus Mendes da Fonseca, ${ }^{1}$ Rosana de Figueiredo França, ${ }^{1}$ \\ Eduardo Faerstein, ${ }^{2}$ Guilherme Loureiro Werneck ${ }^{2}$ e Dóra Chor ${ }^{1}$
}

Como citar Fonseca MJM, França RF, Faerstein E, Werneck GL, Chor D. Escolaridade e padrões de ganho de peso na vida adulta no Brasil: Estudo Pró-Saúde. Rev Panam Salud Publica. 2012;32(5):376-80.

RESUMO Este estudo teve como objetivo investigar a associação entre a escolaridade (considerada como indicador de posição socioeconômica) do respondente e a de seus pais e os padrões de ganho de peso na vida adulta. Foram analisados os dados de 2582 participantes da linha de base (1999) do Estudo Pró-Saúde, investigação longitudinal de funcionários de uma universidade no Rio de Janeiro, Brasil. Utilizaram-se questionários autoadministrados para investigar os padrões de ganho de peso na vida adulta. Foram estimadas as razões de chance (OR) e seus intervalos de confiança de 95\% (IC95\%) para a associação entre escolaridade dos pais e do respondente e aumento de peso constante ou cíclico, tendo o peso estável como referência, por meio de modelos de regressão logística multinomial. Para os homens, menor escolaridade do pai representou chance cerca de $55 \%$ menor de peso cíclico do que de manter o peso constante $(\mathrm{OR}=0,45 ;$ IC95\% = 0,26 a 0,78), enquanto para as mães a escolaridade menor associou-se à maior chance de peso cíclico, embora sem significância estatística $(O R=1,68$; IC95\% =0,94 a 3,00). Não houve associação significativa entre escolaridade do respondente e história de peso entre homens. Entre as mulheres, as de menor escolaridade apresentaram chance $94 \%$ maior de referirem aumento cíclico $(O R=1,94 ;$ IC95\% = 1,17 a 3,23) e não houve associação significativa entre escolaridade dos pais e história de peso. Neste estudo, as variações de peso ao longo da vida, tanto constantes quanto cíclicas, apresentaram associação com a escolaridade dos pais e do próprio indivíduo, com importantes diferenças de gênero em tal associação.

Palavras-chave Peso corporal; ganho de peso; escolaridade; posição socioeconômica; epidemiologia; Brasil.

O aumento da prevalência de obesidade é um dos principais problemas contemporâneos de saúde pública, especialmente pelo aumento do risco de doenças crônicas não transmissíveis (1-3). Características marcadoras da posição socioeconômica (PSE), como escolaridade, têm

\footnotetext{
1 Fundação Oswaldo Cruz, Escola Nacional de Saúde Pública (ENSP/FIOCRUZ), Departamento de Epidemiologia e Métodos Quantitativos em Saúde, Rio de Janeiro (RJ), Brasil. Correspondência: mariajmf@ensp.fiocruz.br (Maria de Jesus Mendes da Fonseca).

2 Universidade do Estado do Rio de Janeiro, Instituto de Medicina Social, Rio de Janeiro (RJ), Brasil.
}

sido associadas à obesidade $(4,5)$. Em uma revisão de 333 artigos, McLaren (6) constatou uma associação inversa entre PSE atual e obesidade entre mulheres de países com índice de desenvolvimento humano (IDH) mais elevado; entretanto, nos países de menor IDH, tal associação era mais frequentemente direta. Para os homens, a maioria dos estudos não evidenciou associação significativa entre PSE e obesidade.

Além disso, sabe-se que existem diferentes padrões de ganho de peso corporal, que variam de acordo com o sexo e o nível de escolaridade $(7,8)$. Os padrões mais frequentes de evolução do peso corporal durante a vida adulta, além da manutenção, são o aumento de peso gradual e o aumento cíclico (também conhecido como peso flutuante ou padrão "ioiô") $(9,10)$. Pressupõe-se que o aumento de peso cíclico acarrete os maiores riscos à saúde, podendo influenciar de forma independente desfechos metabólicos e cardiovasculares, mas as evidências a respeito ainda são inconclusivas (7, 11). No Brasil foram identificados apenas dois estudos - em Porto Alegre e em 
Salvador (nas regiões Sul e Nordeste, respectivamente) - que investigaram a prevalência de aumento de peso cíclico entre pacientes ambulatoriais em tratamento de obesidade, evidenciando aumento cíclico em 13 e $28 \%$ dos pacientes $(9,12)$.

Este trabalho investigou a associação entre escolaridade do participante e de seus pais e padrões de ganho de peso na vida adulta, assim como avaliou possíveis diferenças entre sexos no que diz respeito a essa associação. Trata-se de uma análise dos dados da linha de base (1999) do Estudo Pró-Saúde, uma investigação longitudinal conduzida entre funcionários técnico-administrativos de uma universidade pública no Rio de Janeiro, Brasil. O Pró-Saúde avaliou o papel de marcadores de posição social em diversos aspectos relacionados à saúde. Foram considerados elegíveis todos os funcionários em atividade, sendo inelegíveis apenas os funcionários cedidos a outras instituições ou licenciados por motivos não relacionados à saúde. Participaram 4030 funcionários, representando $91 \%$ do total de elegíveis.

A coleta de dados foi realizada por meio de questionário autoadministrado: 3701 indivíduos relataram o seu padrão de ganho de peso entre os 20 anos de idade e a data do preenchimento do questionário. Para isso, responderam a pergunta "Qual das situações abaixo descreve melhor a evolução do seu peso desde os 20 anos de idade até agora?". Foram oferecidas seis opções de resposta, apresentadas em forma gráfica, indicando manutenção, aumento ou diminuição do peso no período. Para cada uma dessas opções, o indivíduo indicou se a evolução se deu de forma constante ou com oscilações.

As análises aqui apresentadas são baseadas nas respostas de 2582 indivíduos que tinham 30 ou mais anos de idade e apresentaram dados completos para as variáveis estudadas. Foram excluídos das análises os indivíduos com dados incompletos e os que registraram diminuição de peso, já que essa situação teve prevalência abaixo de $2 \%$. Os indivíduos que apresentaram oscilações de peso seja oscilando dentro de uma mesma faixa, seja oscilando com aumento gradativo de peso - foram agrupados em uma única categoria, denominada peso cíclico. Assim, a variável resposta de nosso estudo foi categorizada em: a) aumento constante de peso, b) peso cíclico e c) manutenção do peso constante (categoria de referência).

A confiabilidade foi avaliada em estudo teste-reteste conduzido com 192 indivíduos com características similares às dos participantes do estudo principal. O grau de concordância foi considerado bom, com kappa ponderado de 0,73 (IC95\%: 0,63 a 0,83).

As variáveis utilizadas foram escolaridade dos pais e escolaridade do respondente. A escolaridade dos pais foi categorizada em: menor que o ensino fundamental (EF); EF completo e ensino médio (EM); ou maior do que EM (pelo pequeno número de pais com nível universitário). A escolaridade do respondente foi analisada em três categorias: até EF completo; EM completo; e nível superior (NS) ou mais. Para ambas as variáveis, as categorias de maior nível de escolaridade foram utilizadas como referência nas análises.

As covariáveis avaliadas foram idade, índice de massa corporal (IMC) aos 20 anos e cor/raça. A idade foi considerada de forma categórica na análise bivariada (30 a 34 anos, 35 a 44 anos e >44 anos) e de forma contínua na multivariada. O IMC aos 20 anos de idade, calculado a partir da informação do peso aos 20 anos e da altura aferida no estudo, foi utilizado de forma categórica na análise bivariada (obesidade: $\geq 30 \mathrm{~kg} / \mathrm{m}^{2}$, sobrepeso: 25 a $29,9 \mathrm{~kg} / \mathrm{m}^{2}$ e baixo peso/ eutrófico: $\leq 24,9 \mathrm{~kg} / \mathrm{m}^{2}$ ) e de forma contínua na análise multivariada. A variável cor/raça foi informada pelo participante considerando-se as classes utilizadas pelo Instituto Brasileiro de Geografia e Estatística (branca, parda, preta, amarela e indígena); nas análises foram excluídos os indivíduos autoclassificados como amarelos ou indígenas (2,5\% do total).

A análise univariada enfocou as distribuições de frequências das variáveis do estudo. Na análise bivariada utilizou-se o teste do qui-quadrado de Pearson $\left(\chi^{2}\right)$ para verificar a heterogeneidade da distribuição do desfecho segundo variáveis de exposição e covariáveis. Utilizou-se o valor de $P<0,20$ para seleção das covariáveis a serem incluídas nos modelos multivariados.

$\mathrm{Na}$ análise multivariada utilizou-se o modelo de regressão logística multinomial específica por sexo, com ajuste múltiplo para idade, IMC aos 20 anos e cor/raça. Foram estimadas as razões de chances (odds ratios, OR) e os respectivos intervalos de confiança de 95\% (IC95\%) para a associação entre as variáveis de condição socioeconômica ao longo da vida e o padrão de ganho de peso.

Foram construídos, inicialmente, para ambos os sexos, modelos que continham isoladamente cada uma das variáveis de exposição (escolaridade do pai e da mãe e escolaridade do respondente). Posteriormente, foram acrescentadas as variáveis idade, IMC aos 20 anos e cor/raça. Foram mantidas no modelo final as variáveis que se mostraram significativas $(P<0,05)$ nas análises em pelo menos um dos sexos. Foi utilizado o programa Statistical Package for the Social Sciences (SPSS), versão 17.

O protocolo referente ao Estudo PróSaúde foi aprovado pelo Comitê de Ética da universidade em que foi realizada a pesquisa. A participação foi voluntária e todos os participantes assinaram o termo de consentimento informado.

Dentre os 2582 indivíduos incluídos nas análises, 1160 eram homens (55,1\%) e 1422 mulheres (44,9\%). A média de idade dos homens foi de 40,9 anos (DP = $7,5)$, e das mulheres, de 41,1 anos (DP = $7,3)$. Metade da população encontrava-se na faixa etária de 35 a 44 anos, com 28,4\% acima de 44 anos. Com relação às categorias de padrão de ganho de peso, a prevalência de aumento constante de peso foi maior nos homens $(57,2 \%)$. Entre as mulheres, a maior prevalência foi de peso cíclico $(41,5 \%)$. As prevalências de manutenção de peso constante entre homens e mulheres foram muito próximas - 18,6\% e 19,3\%, respectivamente.

Entre os homens (tabela 1), não houve variações significativas nas prevalências das categorias de padrão de peso segundo escolaridade da mãe, escolaridade do respondente, faixa etária e cor/ raça. A prevalência de peso cíclico entre participantes cujos pais tinham maior escolaridade foi o dobro daquela dos participantes cujos pais tinham fundamental completo. A prevalência de peso cíclico também foi maior entre os que eram obesos aos 20 anos em relação às outras categorias de IMC. Observou-se variação significativa nas prevalências de manutenção de peso constante apenas de acordo com o IMC aos 20 anos, sendo mais frequente a manutenção do peso entre os eutróficos ou com baixo peso.

Entre as mulheres (tabela 1), com exceção da variável escolaridade do pai, observou-se variação significativa nas prevalências das diversas categorias de padrão de ganho de peso. As maiores 
TABELA 1. Padrões de ganho de peso segundo escolaridade dos pais e do respondente e variáveis de ajuste, Estudo Pró-Saúde, Rio de Janeiro, 1999

\begin{tabular}{|c|c|c|c|c|c|c|c|c|}
\hline \multirow[b]{3}{*}{ Variável } & \multicolumn{8}{|c|}{ Prevalência (\%) } \\
\hline & \multicolumn{3}{|c|}{ Homens No. (\%) } & \multirow[b]{2}{*}{$P$-valora } & \multicolumn{3}{|c|}{ Mulheres No. (\%) } & \multirow[b]{2}{*}{$P$-valora } \\
\hline & $\begin{array}{l}\text { Aumento } \\
\text { constante }\end{array}$ & Peso cíclico & Manutenção & & $\begin{array}{l}\text { Aumento } \\
\text { constante }\end{array}$ & Peso cíclico & Manutenção & \\
\hline \multicolumn{9}{|l|}{ Escolaridade do pai } \\
\hline Menor que fundamental & $285(56,9)$ & $117(23,4)$ & $98(19,8)$ & \multirow{3}{*}{0,001} & $266(42,0)$ & $261(41,2)$ & $107(16,9)$ & \multirow{3}{*}{0,068} \\
\hline Fundamental completo & $158(65,6)$ & $37(15,4)$ & $46(19,1)$ & & $107(38,2)$ & $123(43,9)$ & $50(17,9)$ & \\
\hline Ensino médio ou mais & $220(52,6)$ & $127(30,4)$ & $71(17,0)$ & & $185(36,4)$ & $206(40,6)$ & $117(23,0)$ & \\
\hline \multicolumn{9}{|l|}{ Escolaridade da mãe } \\
\hline Menor que fundamental & $340(57,3)$ & $148(25,0)$ & $105(17,7)$ & \multirow{3}{*}{0,562} & $309(41,9)$ & $310(42,0)$ & $119(16,1)$ & \multirow{3}{*}{0,005} \\
\hline Fundamental completo & $147(58,3)$ & $52(20,6)$ & $53(21,0)$ & & $100(33,6)$ & $135(45,3)$ & $63(21,1)$ & \\
\hline Ensino médio ou mais & $276(55,9)$ & $81(25,7)$ & $58(18,4)$ & & $149(38,6)$ & $145(37,6)$ & $92(23,8)$ & \\
\hline \multicolumn{9}{|c|}{ Escolaridade do respondente } \\
\hline Até fundamental & $166(54,6)$ & $78(25,7)$ & $60(19,7)$ & \multirow{3}{*}{0,818} & $91(37,3)$ & $123(50,4)$ & $30(12,3)$ & \multirow{3}{*}{0,0001} \\
\hline Ensino médio completo & $256(57,0)$ & $110(24,5)$ & $83(18,5)$ & & $178(38,6)$ & $214(46,4)$ & $69(15,0)$ & \\
\hline Superior ou mais & $241(59,2)$ & $93(22,9)$ & $73(17,9)$ & & $289(40,3)$ & $253(35,3)$ & $175(24,4)$ & \\
\hline \multicolumn{9}{|l|}{ Faixa etária ${ }^{b}$} \\
\hline 30 a 34 anos & $145(57,5)$ & $54(21,4)$ & $53(21,0)$ & \multirow{3}{*}{0,532} & $106(37,3)$ & $99(34,9)$ & $79(27,8)$ & \multirow{3}{*}{0,0001} \\
\hline 35 a 44 anos & $333(58,0)$ & $137(23,9)$ & $104(18,1)$ & & $268(36,3)$ & $323(43,8)$ & $147(19,9)$ & \\
\hline$>44$ anos & $185(55,4)$ & $90(26,9)$ & $59(17,7)$ & & $184(46,0)$ & $168(42,0)$ & $48(12,0)$ & \\
\hline \multicolumn{9}{|l|}{ IMC aos 20 anos $^{b}$} \\
\hline Obesos & $5(41,7)$ & $6(50,0)$ & $1(8,3)$ & \multirow{3}{*}{0,002} & $8(36,4)$ & $14(63,6)$ & $0(0,0)$ & \multirow{3}{*}{0,001} \\
\hline Sobrepeso & $66(52,4)$ & $45(35,7)$ & $15(11,9)$ & & $20(29,0)$ & $42(60,9)$ & $7(10,1)$ & \\
\hline Baixo peso ou eutrófico & $592(57,9)$ & $230(22,5)$ & $200(19,6)$ & & $530(39,8)$ & $534(40,1)$ & $267(20,1)$ & \\
\hline \multicolumn{9}{|l|}{ Cor/raça ${ }^{b}$} \\
\hline Preta & $83(41,0)$ & $50(30,3)$ & $32(19,4)$ & \multirow{3}{*}{0,174} & $97(38,8)$ & $123(49,2)$ & $30(12,0)$ & \multirow{3}{*}{0,0001} \\
\hline Parda & $211(56,3)$ & $87(23,2)$ & $77(20,5)$ & & $133(35,8)$ & $173(46,5)$ & $66(17,7)$ & \\
\hline Branca & $369(59,5)$ & $144(23,2)$ & $107(17,3)$ & & $328(41,0)$ & $294(36,3)$ & $178(22,3)$ & \\
\hline
\end{tabular}

a Teste do $\chi^{2}, P<0,05$.

b Variáveis de ajuste.

prevalências de aumento constante de peso foram encontradas naquelas cujos pais e mães apresentavam escolaridade mais baixa do que o ensino fundamental. Para as características das respondentes, as maiores prevalências de aumento constante de peso foram para maior escolaridade, idade superior a 44 anos, peso adequado aos 20 anos e cor branca. Com relação ao peso cíclico, as maiores prevalências foram encontradas entre participantes de escolaridade mais baixa, obesas, pretas e cujos pais e a mães tiveram escolaridade de nível fundamental completo.

No modelo final (modelo 2 da tabela 2), entre os homens, observou-se que aqueles cujos pais tinham menor escolaridade apresentaram chance cerca de 55\% menor de peso cíclico do que de manter o peso constante. Já para a mãe, a menor escolaridade associou-se à maior chance de peso cíclico, embora sem alcançar significância estatística. Com relação ao aumento constante de peso, tanto para escolaridade dos pais como do respondente, as associações não foram estatisticamente significativas. Entre as mulheres, não houve associação significativa entre a escolaridade dos pais e o aumento constante de peso ou peso cíclico. Com relação à escolaridade da respondente, houve associação inversa significativa apenas com o peso cíclico. Mulheres com menor escolaridade apresentaram chances quase 2 vezes mais altas de peso cíclico em relação à manutenção do peso constante.

Em nosso estudo, investigou-se a associação entre escolaridade do respondente e de seus pais e padrões de ganho de peso na vida adulta para homens e mulheres. Entre os homens, a menor escolaridade do pai esteve associada a uma menor chance de peso cíclico, mas não ao aumento constante de peso. Neste grupo, a escolaridade da mãe e a do respondente não estiveram associadas aos padrões de ganho de peso na vida adulta. Resultados distintos foram encontrados para as mulheres: a escolaridade dos pais não apresentou associação significativa com os padrões de ganho de peso, mas uma menor escolaridade da respondente esteve associada com maior chance de peso cíclico.

A escolaridade é a variável mais comumente utilizada como indicador de posição socioeconômica (PSE) atual nos estudos sobre o tema $(6,10)$. No caso da
PSE pregressa, os indicadores mais utilizados têm sido a escolaridade dos pais, uma vez que esta influencia fortemente as condições econômicas que o indivíduo vivenciou nas fases iniciais da vida $(8,10,13)$.

Os motivos subjacentes à existência da associação entre menor escolaridade paterna e menor chance de peso cíclico entre homens não são evidentes. Uma hipótese seria de que a escolaridade paterna, estando mais relacionada ao poder aquisitivo da família, representaria um potencial de suprimento de alimentos. Assim, menos recursos financeiros disponíveis levariam à menor chance de peso cíclico pela menor chance de fases de ganho de peso.

Com relação à escolaridade dos respondentes do sexo masculino, não foi encontrada associação com seus padrões de ganho de peso, o que corrobora os achados de outros estudos $(6,14)$. Nesse caso, seria possível pressupor que os homens são menos suscetíveis aos padrões de beleza impostos pela sociedade e que, independentemente de sua escolaridade, seriam mais vulneráveis ao meio obesogênico (caracterizado pela facilidade na aquisição de dietas hipercalóricas e 
TABELA 2. Coeficientes de regressão logística multinomial da associação entre padrões de ganho de peso e indicadores de condição socioeconômica, Estudo Pró-Saúde, Rio de Janeiro, 1999

\begin{tabular}{|c|c|c|c|c|c|c|c|c|}
\hline \multirow[b]{3}{*}{ Variável } & \multicolumn{4}{|c|}{ Homens } & \multicolumn{4}{|c|}{ Mulheres } \\
\hline & \multicolumn{2}{|c|}{$\begin{array}{c}\text { Modelo } 1^{\mathrm{a}} \\
\text { OR (IC95\%) }\end{array}$} & \multicolumn{2}{|c|}{$\begin{array}{c}\text { Modelo } 2^{\mathrm{b}} \\
\text { OR (IC95\%) }\end{array}$} & \multicolumn{2}{|c|}{$\begin{array}{c}\text { Modelo } 1^{\mathrm{a}} \\
\text { OR (IC95\%) }\end{array}$} & \multicolumn{2}{|c|}{$\begin{array}{c}\text { Modelo } 2^{\mathrm{b}} \\
\text { OR (IC95\%) }\end{array}$} \\
\hline & Aumento ${ }^{C}$ & Cíclico ${ }^{C}$ & Aumento ${ }^{C}$ & Cíclico ${ }^{c}$ & Aumento $c$ & Cíclico ${ }^{C}$ & Aumento ${ }^{c}$ & Cíclico ${ }^{C}$ \\
\hline \multicolumn{9}{|l|}{ Escolaridade do pai } \\
\hline Ensino médio ou mais & 1,00 & 1,00 & 1,00 & 1,00 & 1,00 & 1,00 & 1,00 & 1,00 \\
\hline Fundamental completo & $\begin{array}{c}1,09 \\
(0,68 \text { a } 1,73)\end{array}$ & $\begin{array}{c}0,38 \\
(0,22 \text { a } 0,67)\end{array}$ & $\begin{array}{c}1,11 \\
(0,70 \text { a } 1,77)\end{array}$ & $\begin{array}{c}0,39 \\
(0,22 \text { a } 0,70)\end{array}$ & $\begin{array}{c}1,21 \\
(0,78 \text { a } 1,89)\end{array}$ & $\begin{array}{c}1,04 \\
(0,67 \text { a } 1,62)\end{array}$ & $\begin{array}{c}1,25 \\
(0,80 \text { a } 1,95)\end{array}$ & $\begin{array}{c}1,08 \\
(0,69 \text { a } 1,68)\end{array}$ \\
\hline Menor que fundamental & $\begin{array}{c}0,83 \\
(0,52 \text { a } 1,33)\end{array}$ & $\begin{array}{c}0,44 \\
(0,25 \text { a } 0,74)\end{array}$ & $\begin{array}{c}0,86 \\
(0,54 \text { a } 1,38)\end{array}$ & $\begin{array}{c}0,45 \\
(0,26 \text { a } 0,78)\end{array}$ & $\begin{array}{c}1,16 \\
(0,77 \text { a } 1,75)\end{array}$ & $\begin{array}{c}0,88 \\
(0,58 \text { a } 1,32)\end{array}$ & $\begin{array}{c}1,17 \\
(0,54 \text { a } 1,38)\end{array}$ & $\begin{array}{c}0,85 \\
(0,56 \text { a } 1,27)\end{array}$ \\
\hline \multicolumn{9}{|l|}{ Escolaridade da mãe } \\
\hline Ensino médio ou mais & 1,00 & 1,00 & 1,00 & 1,00 & 1,00 & 1,00 & 1,00 & 1,00 \\
\hline Fundamental completo & $\begin{array}{c}0,95 \\
(0,59 \text { a } 1,52)\end{array}$ & $\begin{array}{c}0,97 \\
(0,56 \text { a } 1,69)\end{array}$ & $\begin{array}{c}0,97 \\
(0,61 \text { a } 1,56)\end{array}$ & $\begin{array}{c}0,92 \\
(0,53 \text { a } 1,62)\end{array}$ & $\begin{array}{c}0,85 \\
\left(0,55^{\mathrm{a}} 1,31\right)\end{array}$ & $\begin{array}{c}1,16 \\
(0,75 \text { a } 1,77)\end{array}$ & $\begin{array}{c}0,79 \\
(0,50 \text { a } 1,22)\end{array}$ & $\begin{array}{c}1,03 \\
(0,66 \text { a } 1,59)\end{array}$ \\
\hline Menor que fundamental & $\begin{array}{c}1,29 \\
(0,76 \text { a } 2,10)\end{array}$ & $\begin{array}{c}1,74 \\
(0,99 \text { a } 3,06)\end{array}$ & $\begin{array}{c}1,34 \\
(0,82 \text { a } 2,19)\end{array}$ & $\begin{array}{c}1,68 \\
(0,94 \text { a } 3,00)\end{array}$ & $\begin{array}{c}1,28 \\
(0,84 \text { a } 1,94)\end{array}$ & $\begin{array}{c}1,30 \\
(0,85 \text { a } 1,99)\end{array}$ & $\begin{array}{c}1,18 \\
(0,77 \text { a } 1,80)\end{array}$ & $\begin{array}{c}1,81 \\
(0,77 \text { a } 1,82)\end{array}$ \\
\hline \multicolumn{9}{|l|}{ Escolaridade do respondente } \\
\hline Superior ou mais & 1,00 & 1,00 & 1,00 & 1,00 & 1,00 & 1,00 & 1,00 & 1,00 \\
\hline Ensino médio completo & $\begin{array}{c}0,91 \\
(0,62 \text { a } 1,32)\end{array}$ & $\begin{array}{c}1,09 \\
(0,70 \text { a } 1,68)\end{array}$ & $\begin{array}{c}0,97 \\
(0,66 \text { a } 1,41)\end{array}$ & $\begin{array}{c}1,15 \\
(0,73 \text { a } 1,80)\end{array}$ & $\begin{array}{c}1,45 \\
(1,03 \text { a } 2,05)\end{array}$ & $\begin{array}{c}2,08 \\
(1,48 \text { a } 2,94)\end{array}$ & $\begin{array}{c}1,35 \\
(0,95 \text { a } 1,93)\end{array}$ & $\begin{array}{c}1,85 \\
(1,29 \text { a } 2,64)\end{array}$ \\
\hline Até fundamental & $\begin{array}{c}0,81 \\
(0,53 \text { a } 1,25)\end{array}$ & $\begin{array}{c}1,14 \\
(0,69 \text { a } 1,88)\end{array}$ & $\begin{array}{c}0,87 \\
(0,56 \text { a } 1,36)\end{array}$ & $\begin{array}{c}1,12 \\
(0,66 \text { a } 1,91)\end{array}$ & $\begin{array}{c}1,52 \\
(0,94 \text { a } 2,46)\end{array}$ & $\begin{array}{c}2,71 \\
(1,69 \text { a } 4,35)\end{array}$ & $\begin{array}{c}1,09 \\
(0,65 \text { a } 1,83)\end{array}$ & $\begin{array}{c}1,94 \\
(1,17 \text { a } 3,23)\end{array}$ \\
\hline
\end{tabular}

a Modelo 1: escolaridade do pai + escolaridade da mãe + escolaridade do respondente.

${ }^{b}$ Modelo 2: escolaridade do pai + escolaridade da mãe + escolaridade do respondente + idade + IMC aos 20 anos + raça.

c Aumento = aumento de peso constante desde os 20 anos; cíclico = aumento de peso cíclico desde os 20 anos.

pela diminuição nos níveis de atividade física). Assim, para eles, a escolaridade não contribuiria para a mudança de hábitos alimentares adquiridos na infância $(6,14,15)$.

Entre as mulheres, a menor escolaridade associou-se à maior chance de peso cíclico. Esse padrão pode ser um reflexo da diferença de acesso à informação e a medidas de controle de peso, mais ao alcance das mulheres com maior escolaridade. Ainda assim, foram observadas prevalências mais altas de peso cíclico em quase todas as categorias de escolaridade, sugerindo maior frequência de utilização, pelas mulheres, de estratégias para redução de peso, possivelmente pela preocupação mais acentuada com a saúde e com a adequação aos atuais padrões de beleza $(13,14)$.

Segundo McLaren (6), a associação inversa entre PSE e ganho de peso entre as mulheres, nos países desenvolvidos, retrata a valorização e a dedicação à magreza nas camadas sociais mais altas. Demonstra ainda um gradiente socioeconômico relacionado também à dieta, pelo qual pessoas com melhor condição socioeconômica apresentam dietas mais saudáveis, caracterizadas pelo consumo de frutas e hortaliças em maior variedade, assim como de produtos dietéticos e com menor teor de gordura. Esse padrão de consumo reflete, também, a capacidade econômica de compra, pois, em geral, esses dois últimos tipos de alimentos são mais caros.

É importante notar, no contexto do presente estudo, que as definições utilizadas para peso cíclico na literatura, assim como os valores que o definem, não são consensuais. Os valores utilizados para definir perda de peso variam entre 4,5 e 9 $\mathrm{kg}$ por ciclo, ou perda de $2 \mathrm{~kg}$ no período de 5 anos, ou ainda perda de $5 \%$ do peso entre outros. Neste estudo, entretanto, o critério utilizado foi o autorrelato baseado em esquemas gráficos que mostravam diferentes padrões de evolução do peso a partir dos 20 anos de idade. Ainda que este método de aferição possa ser menos acurado, a abordagem gráfica facilita que o respondente se enquadre em uma trajetória mais geral de evolução de peso, eventualmente atenuando erros relacionados à necessidade de recordação exata do peso em diferentes momentos no passado.

Associações inconsistentes entre escolaridade e peso cíclico podem derivar de variações nas prevalências de obesidade nas amostras utilizadas em diferentes estudos. Além disso, como o peso cíclico é caracterizado como um processo em que há repetidas perdas e ganhos de peso, pode ser mais fácil identificar esses pa- drões entre pessoas com sobrepeso ou obesidade, por fazerem dietas mais frequentemente (11).

Outra questão a ser considerada nessas análises é a possibilidade de que os resultados sejam influenciados pela estrutura etária da população estudada. Por exemplo, a inclusão de pessoas muito jovens que ainda não tiveram tempo de atingir o ápice da primeira onda cíclica poderia dificultar a identificação desse padrão específico de evolução do peso. Neste estudo, procurou-se minimizar esse problema pelo ajuste para a idade nas análises multivariadas e pela inclusão apenas indivíduos com idade maior do que 30 anos.

Outra preocupação diz respeito à possibilidade de um efeito de geração. Por exemplo, a escolaridade dos pais dos indivíduos mais jovens pode ser maior, em média, do que a escolaridade dos pais dos participantes com mais de 50 anos. Para verificar tal possibilidade, realizou-se análise estratificada utilizando a média de idade (41 anos) para definir os estratos. Os resultados encontrados foram similares aos da análise não estratificada.

O fato de termos analisado uma coorte de funcionários públicos limita a comparação de nossos resultados com aqueles baseados na população geral. Entretanto, 
essa população apresenta relativa heterogeneidade demográfica e socioeconômica, similar à encontrada nas camadas médias urbanas do país.

Analisando os diferentes desfechos relacionados aos padrões de ganho de peso em homens e mulheres, pode-se observar que, independentemente do IMC aos 20 anos de idade, as variações ponderais que ocorrem ao longo da vida, sejam elas constantes ou cíclicas, apresentam asso- ciação com a escolaridade dos pais e do próprio indivíduo, com importantes diferenças de gênero em tal associação.

Nossos resultados corroboram as evidências apresentadas em estudos internacionais no sentido da existência de associação entre escolaridade atual e dos pais e os padrões de ganho de peso $(3,10)$. Essa associação sugere o início precoce de desigualdades de saúde que podem se manifestar na vida adulta.
Além disso, sugere que a atual epidemia de obesidade, assim como os agravos dela decorrentes, deve ser analisada observando-se a trajetória de peso das pessoas.

Agradecimentos. O estudo recebeu financiamento do Conselho Nacional de Desenvolvimento Científico e Tecnológico (CNPq) (processo 302322/2002-7).

\section{REFERÊNCIAS}

1. World Health Organization. Overweight and obesity. Genebra: WHO; 2011. (Fact sheet no. 311).

2. Guh DP, Zhang W, Bansback N, Amarsi Z, Birmingham CL, Anis AH. The incidence of co-morbidities related to obesity and overweight: a systematic review and meta-analysis. BMC Public Health. 2009;9:88.

3. Alley DE, Chang VW. Metabolic syndrome and weight gain in adulthood. J Gerontol A Biol Sci Med Sci. 2010;65(1):111-7.

4. Faeh D, Braun J, Bopp M. Prevalence of obesity in Switzerland 1992-2007: the impact of education, income and occupational class. Obes Rev. 2011;12(3):151-66.

5. Sichieri R, Moura EC. Análise multinível das variações no índice de massa corporal entre adultos, Brasil, 2006. Rev Saude Publica. 2009;43(Suppl 2):90-7.

6. McLaren L. Socioeconomic status and obesity. Epidemiol Rev. 2007;29(1):29-48. doi:10.1093/ epirev/mxm001 PMID:17478442

7. Andrade BMC, Mendes CMC, Araújo LMB. Peso flutuante no tratamento de mulheres obesas. Arq Bras Endocrinolol Metab. 2004; 48(2):276-81.

8. Ball K, Crawford D. Socioeconomic status and weight change in adults: a review. Soc Sci Med. 2005;60(9):1987-2010. doi:10.1016/ j.socscimed.2004.08.056

9. Montani J-P, Viecelli AK, Prévot A, Dulloo AG. Weight cycling during growth and beyond as a risk factor for later cardiovascular diseases: the 'repeated overshoot' theory. Int J Obes (Lond). 2006;30(Suppl 4);S58-66.

10. Schulz M, Liese AD, Boeing $H$, Cunningham JE, Moore CG, Kroke A. Associations of shortterm weight changes and weight cycling with incidence of essential hypertension in the EPIC-Potsdam Study. J Hum Hypertens. 2005;19(1):61-7.

11. Ball K, Mishra GD. Whose socioeconomic status influences a woman's obesity risk: her mother's, her father's, or her own? Int J Epidemiol. 2006;35(1):131-8.

12. Oliveira SPSG, Pecis M, Aveline MK, Schmidt AC, Zaslavsky LMA, Souto KEP, et al. Flutuação de peso nos pacientes atendidos no ambu- latório de endocrinologia do Hospital Nossa Senhora da Conceição entre 2002 e 2005. Rev AMRIGS. 2007;51(3):190-7.

13. Sobal J, Stunkard AJ. Socioeconomic status and obesity: a review of the literature. Psychol Bull. 1989;105(2):260-75.

14. da Fonseca MJ, Faerstein E, Chor D, Lopes CS, Andreozzi VL. Associações entre escolaridade, renda e índice de massa corporal em funcionários de uma universidade no Rio de Janeiro, Brasil: estudo Pró-Saúde. Cad Saude Publica. 2006;22(11):2359-67.

15. Monteiro CA, Conde WL, Popkin BM. Incomespecific trends in obesity in Brazil: 1975-2003. Am J Public Health. 2007;97(10):1808-12.

Manuscrito recebido em 27 de outubro de 2011. Aceito em versão revisada em 30 de julho de 2012.
ABSTRACT

\section{Educational status and patterns of weight gain in adulthood in Brazil: Estudo Pró-Saúde}

Key words
The aim of the present study was to investigate the association between participant and parental educational status (considered as an indicator of socioeconomic status) and participant pattern of weight gain in adulthood. We analyzed data from 2582 baseline participants (1999) of Estudo Pró-Saúde (Pro-Health Study), a longitudinal investigation of civil servants from a public university in Rio de Janeiro, Brazil. Self-administered questionnaires were used to identify patterns of weight gain in adulthood. Odds ratios (OR) and 95\% confidence intervals $(95 \% \mathrm{CI})$ were estimated for the association between parental and participant educational status and steady weight gain or weight cycling, with stable weight as a reference, using multinomial logistic regression models. For males, lower paternal educational level entailed a chance about $55 \%$ lower of weight cycling as compared to stable weight $(\mathrm{OR}=0.45$; IC95\% $=0.26-0.78)$, whereas lower maternal schooling was related to increased risk of weight cycling, although without reaching statistical significance $(\mathrm{OR}=1.68$; IC95\% $=$ 0.94-3.00). The association between participant educational status and weight history was not statistically significant among men. In women, lower educational status entailed a chance $94 \%$ higher of self-reported weight cycling $(\mathrm{OR}=1.94 ; 95 \% \mathrm{CI}=$ 1.17-3.23), and there was no association between parental educational level and history of weight gain. In this study, changes in weight throughout life, both steady and cyclic, were associated with parental and participant educational status, with major differences between genders.

Body weight; weight gain; social class; educational status; epidemiology; Brazil. 